\title{
Author Index Volume 34 (2014)
}

The issue number is given in front of the pagination

Abantanga, F., see Scherber, S. (4) 139-149

Abd El hafez, A. and A. El-aty Shawky, Analysis of metaplastic breast carcinoma: FNAC; histopathology and immunohistochemistry are complementary for diagnosis (2) 67-75

AbdullGaffar, B., E. Ghazi, E. Mohamed and D. Hamza, Breast metaplastic carcinoma with unusual small cell component (1) 19-24

Adane, S., see Cherbal, F. (1) 1-8

Adjei, E., see Scherber, S. (4) 139-149

Aggarwal, L., see Suhani (3) 135-138

Aggarwal, L., see Suhani (3) 95-99

Ahmed, A.A.M., Clinicopathological profile of female sudanese patients with locally advanced breast cancer (3) 131-134

Alawad, A.A.M., Multiple parenchymal leiomyomas of the breast in a Sudanese female (4) 165-167

Ali, S., see Suhani (3) 135-138

Ali, S., see Suhani (3) 95-99

Anthony, P., see Khandelwal, R. (2) 57-59

Anthony, P., see Khandelwal, R. (4) 151-155

Atares, M., see Blay, J. (1) 43-46

Awuah, B., see Scherber, S. (4) 139-149

Ayed, C.B., see Charfi, S. (2) 87-93

Ayyappan, A.P., see Gaur, S. (2) 81-86

Bahadur, S., M. Pujani, S. Jetley and P.K. Raina, Mucinous Carcinoma of Breast with Psammomatous calcification: Report of a rare case with extensive axillary metastases (4) 177-181

Bakour, R., see Cherbal, F. (1) 1-8

Bezić, J., I. Mrklić, Z. Pogorelić and S. Tomić, Mammographic screening has failed to improve pathohistological characteristics of breast cancers in Split region of Croatia (2) 47-51

Blay, J., R. Medina, N. Rausell, C. Fonfria, M. Atares, L. Requeni and J. Vilar, Unilateral mastitis obliterans presented as a palpable breast mass in a patient with long-standing diabetes mellitus (1) 43-46
Boualga, K., see Cherbal, F. (1) 1-8

Boudawara-Sellami, T., see Charfi, S. (2) 87-93

Chaabane, K., see Charfi, S. (2) 87-93

Charfi, S., C.B. Ayed, H. Mnif, S. Ellouze, K. Chaabane, J. Feki, M. Frikha, J. Daoud and T. Boudawara-Sellami, Mammary neuroendocrine carcinoma with mucinous differentiation: A clinicopathological study of 15 cases (2) 87-93

Cherbal, F., R. Bakour, S. Adane and K. Boualga, BR$C A 1$ and BRCA2 germline mutation spectrum in hereditary breast/ovarian cancer families from Maghrebian countries (1) 1-8

Chintamani, see Tandon, M. (3) 127-130

Choudhary, M., see Suhani (3) 135-138

Christakis, M., see Galyfos, G. (4) 169-172

Civitelli, L., see Rossi, L. (4) 173-176

Daoud, J., see Charfi, S. (2) 87-93

Dedicoat, M., see Green, M. (1) 39-42

Deligiannis, E., see Galyfos, G. (4) 169-172

Deniz, S., E. Vardar, R. Öztürk, İ. Zihni, A. Yağcı and F. Taşlı, Pseudo-angiomatous stromal hyperplasia of the breast detecting in mammography. Case report and review of the literature (3) 117-120

Desai, G., see Suhani (3) 95-99

Deswal, V., see Suhani (3) 135-138

Dolas, C.S., see Poovamma, C.U. (3) 101-104

Dolas, S.C., C.U. Poovamma, M. Prema, R. Khandelwal, A.V. Pais and A. Kaul, Poland's Syndrome: A case report with review of literature regarding management (3) 121-125

El-aty Shawky, A., see Abd El hafez, A. (2) 67-75

Ellouze, S., see Charfi, S. (2) 87-93

Feki, J., see Charfi, S. (2) 87-93

Feldman, E., see Sosin, M. (1) 35-38

Fonfria, C., see Blay, J. (1) 43-46 
Frikha, M., see Charfi, S. (2) 87-93

Funder, J.A., see Mele, M. (4) 183-187

Galyfos, G., G. Karantzikos, A. Sianou, D. Karatzia, M. Christakis and E. Deligiannis, Squamous cell carcinoma of the hand metastasized to the breast: A unique case (4) 169-172

Gaur, S., A.P. Ayyappan and Z. Nahleh, Breast metastases from an adrenocorticotropic hormone secreting thymic neuro-endocrine tumor (2) 81-86

Ghazi, E., see AbdullGaffar, B. (1) 19-24

Gierach, G.L., see Sherman, M.E. (3) 105-116

Green, M., E. Millar, H. Merai, M. O'Shea, M. Dedicoat and $\mathrm{H}$. Ingle, Mammary Tuberculosis in the young: A case report and literature review (1) 39-42

Hamza, D., see AbdullGaffar, B. (1) 19-24

Ingle, H., see Green, M. (1) 39-42

Jain, I., see Khandelwal, R. (2) 53-55

Jain, M., see Suhani (3) 95-99

Jensen, V., see Mele, M. (4) 183-187

Jetley, S., see Bahadur, S. (4) 177-181

Kantounakis, I., see Salemis, N.S. (2) 61-65

Karantzikos, G., see Galyfos, G. (4) 169-172

Karatzia, D., see Galyfos, G. (4) 169-172

Kaul, A., see Dolas, S.C. (3) 121-125

Kawai, J., see Ooe, A. (1) 9-17

Khandelwal, R. and I. Jain, Breast tuberculosis mimicking a malignancy: A rare case report with review of literature (2) 53-55

Khandelwal, R., C.U. Poovamma, C. Shilpy, M. Prema and P. Anthony, Axillary reverse mapping: Is it feasible in locally advanced breast cancer patients? (4) 151-155

Khandelwal, R., C.U. Poovamma, C. Shilpy, M. Prema and $\mathrm{P}$. Anthony, Breast cancer presenting in the midline without a lesion in the breast: A therapeutic dilemma (2) 57-59

Khandelwal, R., see Dolas, S.C. (3) 121-125

Khandelwal, R., see Poovamma, C.U. (3) 101-104

Knight, V., see Morris, O.J. (4) 157-163

Kumar, J., see Suhani (3) 95-99

Logan, D., see Morris, O.J. (4) 157-163

Medina, R., see Blay, J. (1) 43-46

Meena, K., see Suhani (3) 95-99
Mele, M., P. Vahl, J.A. Funder, A.S. Sorensen and V. Jensen, Apocrine Carcinoma arising in a Complex Fibroadenoma: A case report (4) 183-187

Merai, H., see Green, M. (1) 39-42

Merajver, S.D., see Scherber, S. (4) 139-149

Mies, C., see Sherman, M.E. (3) 105-116

Millar, E., see Green, M. (1) 39-42

Mnif, H., see Charfi, S. (2) 87-93

Mohamed, E., see AbdullGaffar, B. (1) 19-24

Morris, O.J., V. Knight and D. Logan, Intra-operative ultrasound versus wire-guided localization in the surgical management of non-palpable breast cancer (4) 157-163

Mrklić, I., see Bezić, J. (2) 47-51

Nahleh, Z., see Gaur, S. (2) 81-86

Nakos, G., see Salemis, N.S. (1) 29-34

Nakos, G., see Salemis, N.S. (2) 61-65

Nangia, A., K. Patiri, M. Pujani and S. Sehgal, Bilateral Myoid Hamartoma of breast: An exceptionally rare lesion (2) 77-80

Nisheena, R., see Poovamma, C.U. (3) 101-104

Ooe, A., S. Takahara, K. Sumiyoshi, H. Yamamoto, J. Kawai and E. Shiba, Relationship between intrinsic subtypes and tumor responses to neoadjuvant chemotherapy in patients with locally advanced breast cancer (1) 9-17

Osei-Bonsu, E., see Scherber, S. (4) 139-149

O'Shea, M., see Green, M. (1) 39-42

Öztürk, R., see Deniz, S. (3) 117-120

Pais, A.V., see Dolas, S.C. (3) 121-125

Pais, V.A., see Poovamma, C.U. (3) 101-104

Panwar, P., see Tandon, M. (3) 127-130

Patiri, K., see Nangia, A. (2) 77-80

Pogorelić, Z., see Bezić, J. (2) 47-51

Poovamma, C.U., see Dolas, S.C. (3) 121-125

Poovamma, C.U., see Khandelwal, R. (2) 57-59

Poovamma, C.U., see Khandelwal, R. (4) 151-155

Poovamma, C.U., V.A. Pais, C.S. Dolas, M. Prema, R. Khandelwal and R. Nisheena, Idiopathic granulomatous mastitis: A rare entity with a variable presentation (3) 101-104

Prema, M., see Dolas, S.C. (3) 121-125

Prema, M., see Khandelwal, R. (2) 57-59

Prema, M., see Khandelwal, R. (4) 151-155

Prema, M., see Poovamma, C.U. (3) 101-104

Pujani, M., see Bahadur, S. (4) 177-181

Pujani, M., see Nangia, A. (2) 77-80 
Raina, P.K., see Bahadur, S. (4) 177-181

Ranieri, E., see Rossi, L. (4) 173-176

Rausell, N., see Blay, J. (1) 43-46

Requeni, L., see Blay, J. (1) 43-46

Rossi, L., S. Zancla, L. Civitelli and E. Ranieri, An unusual orbital metastasis of breast cancer (4) 173176

Salemis, N.S., Breast hematoma complicating anticoagulant therapy: Management and literature review (1) 25-28

Salemis, N.S., C. Seretis, G. Nakos, I. Kantounakis, C. Stoumpos and K. Spiliopoulos, Synchronous occurrence of breast cancer and pulmonary sclerosing hemangioma: Management and review of the literature (2) 61-65

Salemis, N.S., G. Nakos, E. Tsiambas, V. Tsantilas and C. Seretis, Rapidly growing myofibroblastoma of the breast diagnosed in a premenopausal woman: Management and review of the literature (1) 2934

Scherber, S., A.S. Soliman, B. Awuah, E. Osei-Bonsu, E. Adjei, F. Abantanga and S.D. Merajver, Characterizing breast cancer treatment pathways in $\mathrm{Ku}$ masi, Ghana from onset of symptoms to final outcome: Outlook towards cancer control (4) 139149

Sehgal, S., see Nangia, A. (2) 77-80

Seretis, C., see Salemis, N.S. (1) 29-34

Seretis, C., see Salemis, N.S. (2) 61-65

Sherman, M.E., C. Mies and G.L. Gierach, Opportunities for molecular epidemiological research on ductal carcinoma in-situ and breast carcinogenesis: Interdisciplinary approaches (3) 105-116

Shiba, E., see Ooe, A. (1) 9-17

Shilpy, C., see Khandelwal, R. (2) 57-59

Shilpy, C., see Khandelwal, R. (4) 151-155

Sianou, A., see Galyfos, G. (4) 169-172
Soliman, A.S., see Scherber, S. (4) 139-149

Sorensen, A.S., see Mele, M. (4) 183-187

Sosin, M. and E. Feldman, Giant juvenile fibroadenoma: A case and review of novel modalities in treatment (1) 35-38

Spiliopoulos, K., see Salemis, N.S. (2) 61-65

Stoumpos, C., see Salemis, N.S. (2) 61-65

Suhani, S. Ali, G. Desai, S. Thomas, L. Aggarwal, K. Meena, J. Kumar, M. Jain and S.K. Tudu, Primary neuroendocrine carcinoma breast: Our experience (3) 95-99

Suhani, S. Thomas, S. Ali, M. Choudhary, V. Deswal and L. Aggarwal, Denovo malignant fibrous histiocytoma of breast: Report of a case and review of literature (3) 135-138

Sumiyoshi, K., see Ooe, A. (1) 9-17

Taşl1, F., see Deniz, S. (3) 117-120

Takahara, S., see Ooe, A. (1) 9-17

Tandon, M., Chintamani and P. Panwar, Breast Tuberculosis at a tertiary care centre: A retrospective analysis of 22 cases (3) 127-130

Thomas, S., see Suhani (3) 135-138

Thomas, S., see Suhani (3) 95-99

Tomić, S., see Bezić, J. (2) 47-51

Tsantilas, V., see Salemis, N.S. (1) 29-34

Tsiambas, E., see Salemis, N.S. (1) 29-34

Tudu, S.K., see Suhani (3) 95-99

Vahl, P., see Mele, M. (4) 183-187

Vardar, E., see Deniz, S. (3) 117-120

Vilar, J., see Blay, J. (1) 43-46

Yağc1, A., see Deniz, S. (3) 117-120

Yamamoto, H., see Ooe, A. (1) 9-17

Zancla, S., see Rossi, L. (4) 173-176

Zihni, İ., see Deniz, S. (3) 117-120 